De quelques enjeux et usages historiques du Français fondamental

\title{
Les débats sur le Français fondamental et leur influence sur l'enseignement du français en Allemagne
}

Herbert Christ et Ingeborg Christ

\section{(2) OpenEdition}

Édition électronique

URL : https://journals.openedition.org/dhfles/1199

DOI : 10.4000/dhfles. 1199

ISSN : 2221-4038

Éditeur

Société Internationale pour l'Histoire du Français Langue Étrangère ou Seconde

Édition imprimée

Date de publication : 1 janvier 2006

Pagination : 153-172

ISSN : 0992-7654

Référence électronique

Herbert Christ et Ingeborg Christ, « Les débats sur le Français fondamental et leur influence sur l'enseignement du français en Allemagne », Documents pour l'histoire du français langue étrangère ou seconde [En ligne], 36 | 2006, mis en ligne le 06 septembre 2011, consulté le 27 mai 2021. URL : http:// journals.openedition.org/dhfles/1199; DOI : https://doi.org/10.4000/dhfles.1199

Ce document a été généré automatiquement le 27 mai 2021.

(c) SIHFLES 


\title{
Les débats sur le Français fondamental et leur influence sur l'enseignement du français en Allemagne
}

\author{
Herbert Christ et Ingeborg Christ
}

\section{L'enseignement du français des années 1950 dans les écoles et les « Universités populaires » allemandes}

1 En République fédérale d'Allemagne, le français langue étrangère occupe, dans les années 1950, aussi bien dans les lycées et les collèges que dans les Universités populaires (les « Volkshochschulen », institutions d'enseignement pour les adultes) une confortable deuxième place, après l'anglais. En République démocratique allemande, on constate pendant cette période, une prédominance du russe, les autres langues étant marginalisées.

2 On enseignait - en RFA et RDA - la langue écrite, voire littéraire. La finalité de l'enseignement était d'aboutir, dans les grandes classes et dans les universités, aux études littéraires et de civilisation. Les classes de débutants avaient la fonction de préparer aux grandes classes, on ne leur accordait pas une valeur propre. La méthodologie prédominante pour les cours de débutants était, selon les programmes officiels, " la méthode directe ", ou une " méthode éclectique " ${ }^{1}$ - la " vermittelnde Methode ». On faisait semblant d'enseigner la langue parlée, en pratiquant une langue écrite oralisée. 


\section{L'enseignement du français sous l'influence du Français fondamental}

3 L'enseignement du français a changé sous l'influence du Français fondamental. Un programme officiel, le "Curriculum " pour le second cycle de l'enseignement secondaire du Land Rhénanie du Nord -Westphalie ${ }^{2}$ résume, quelques années plus tard, cette transformation (Kultusministerium 1973). On y distingue deux degrés d'enseignement du français : un premier degré d'une durée de 2 ans (qui ressemble au " Niveau I ») $)^{3}$, et un deuxième degré relativement ouvert quant à la durée, qui ressemble au « Niveau II » avec des options pour un « Niveau III » des langues de spécialité.

4 Au premier degré, la priorité est accordée à la langue parlée, sans exclure cependant la langue écrite.

Le choix du lexique que l'on enseigne aux élèves se fera selon les principes d'un

vocabulaire fondamental français (fréquence, disponibilité, progression). L'enseignement de la grammaire, centré sur la morphosyntaxe, respectera ces principes linguistiques et pédagogiques (Kultusministerium $1973: 26$. Traduit par nous).

5 Le programme préconise " les principes d'un vocabulaire fondamental français ». L'élève devra acquérir, dans les deux premières années, environ 250 mots "grammaticaux» et 1500 mots «lexicaux», un vocabulaire qui dépasse numériquement celui du Français fondamental premier degré (FF1). On lui explique la différence entre l'oral et l'écrit (quant à la grammaire et au lexique), et il apprendra à distinguer entre l'expression orale (relativement) spontanée et l'expression écrite, plutôt réfléchie. L'enseignement de la grammaire sera centré sur la morphosyntaxe. On respectera là aussi les principes de fréquence, de disponibilité et de progression, qui ont déterminé le choix de la « grammaire » du FF1.

$6 \mathrm{Au}$ second degré - préparé par une phase intermédiaire ("Übergangsstufe ») dans laquelle on s'occupe spécialement du passage de l'oral à l'écrit - on enseignera la « langue soignée » (selon la terminologie de C Stourdzé (1969). Le vocabulaire est développé selon les programmes spécifiques des classes. Ceci veut dire que l'on n'enseignera ni le vocabulaire ni la grammaire du Français fondamental du deuxième degré (FF2) tels quels, et pas non plus un vocabulaire et une grammaire uniformes pour toutes les classes. On adopte cependant certains résultats des travaux concernant le FF2, notamment le concept de centres d'intérêt, et la progression en " Niveaux " superposés. Le Français fondamental a laissé ses traces dans les programmes officiels, sans être accepté tel quel.

7 L'enseignement des langues dans les Volkshochschulen était dans une autre situation que dans les lycées et collèges. Ces établissements autonomes ne connaissaient ni programmes officiels, ni examens à la fin des cours, et pas non plus une formation spéciale des professeurs. Dans les années 1950, chaque professeur enseignait en principe à sa guise, dans la plupart des cas à l'instar de l'enseignement des lycées. Mais dès les années soixante, des didacticiens, engagés par les fédérations des Volkshochschulen (les Landesverbände), commençaient à définir et à standardiser l'offre. On concevait des examens - baptisés Zertifikat -dans l'intention d'homogénéiser les programmes, pour ainsi dire " à rebours ». La commission du VHS-Zertifikat Französisch s'appuyait sur le FF1 pour décrire les contenus langagiers (Raasch 1969a, 1969b, 1977 ; Deutscher Volkshochschul-Verband e.V. 1979). La conception du Français 
fondamental a même inspiré les commissions pour d'autres langues (cf. Barrera-Vidal 1977, Kaufmann 1977, Oehler 1972, Reiske 1977).

\section{La réception du Français fondamental en linguistique et en didactique des langues}

8 Avant l'acceptation et l'application du Français fondamental par les administrations des Ecoles et les Fédérations des Volkshochschulen, il y avait eu de longues discussions en didactique et en linguistique. On trouve un excellent résumé de ces débats dans un livre de Harro Stammerjohann (1983 : 161-212). Il souligne la longue tradition dans laquelle les discussions s'inscrivent : le critère de la fréquence est discuté depuis les recherches de Friedrich-Wilhelm Kaeding (1898), le critère de la répartition a été introduit dans la discussion par George Vander Beke en 1929, le critère de la disponibilité est discuté depuis les années 30 sous le terme allemand Gebrauchswert (Bakonij 1934).

9 Voici quelques points des débats allemands sur le Français fondamental :

10 - Il y avait unanimité parmi les chercheurs sur le rôle accordé à la langue parlée.

11 - On saluait en plus le Français fondamental comme instrument de description d'une langue ouverte (et non pas - à la manière du BASIC - d'une langue close).

12 - On critiquait le purisme " académique » de la commission qui avait supprimé l'oralité authentique en faveur d'une langue châtiée.

13 - On critiquait également le choix des locuteurs interviewés (leur répartition selon l'âge, la région, l'état social, cf. Pfeffer 1969), et leur manque de représentativité.

14 - Un autre point de la critique était la délimitation des « mots » (qu'est-ce qu'un " mot » pour les rédacteurs du Français fondamental ?). On s'étonne de la notation uniforme des mots : les verbes à l'infinitif, les noms au singulier, etc., sans tenir compte des formes fréquentes.

15 - On critique le fait que les « mots » apparaissent sans leur contexte, ce qui est surtout fatal dans le cas des homonymes. On critique en plus la compilation du vocabulaire oral " disponible » par une enquête à base de listes de mots écrites.

16 - Certains critiques posent la question de savoir si l'on peut séparer strictement langue parlée et langue écrite, en théorie comme en pratique, comme prétend le faire la commission du Français fondamental, et on se demande s'il est prudent d'essayer de le faire en classe de langue, sans discuter la finalité de l'enseignement dans le curriculum (Nickolaus 1972a).

17 - On souligne que le Français fondamental - qui est le résultat d'une recherche empirique bien déterminée dans l'espace et le temps - ne peut pas être appliqué sans modifications dans tous les contextes, sans tenir compte de la situation et des besoins des apprenants.

18 - On préfère clairement le terme « fondamental » à la notion « élémentaire ». La notion "fondamental " est traduite en allemand par le lemme "Grund ", dans des mots composés comme «Grundwortschatz» (Nickolaus 1961, Christ 1970, Oehler 1972, Betz et Stammerjohann 1975, Abel 1976) ou «Grunddeutsch» ${ }^{4}$ (Pfeffer 1969, Stötzel 1970). Certains chercheurs préfèrent d'autres lemmes comme par exemple "Basis » (cf. " Basiswortschatz », Tarnöczi 1971) , « Mindest » (cf. « Mindestwortschatz », Raasch 
1969a) ou « Minimal » («Minimalwortschatz», Hüllen et al. 1977). Les lemmes « Minimal » et « Mindest » modifient, il est vrai, le concept de « fondamental ».

\section{Les matériaux pour la classe élaborés sous l'influence du Français fondamental : le cas exemplaire du manuel Salut}

19 Nous arrêtons ici ce bref survol sur la discussion théorique du Français fondamental, pour nous pencher sur son application en classe de français en Allemagne. Faute de disposer de dates empiriques ${ }^{6}$, nous allons étudier un manuel qui est conçu et rédigé selon les principes du Français fondamental. Ce manuel, Salut (Barrera-Vidal et Franke 1968a), eut dès sa parution un grand succès. Les maisons d'édition concurrentes en ont tiré des conséquences, et on peut dire que Salut a donné naissance à une nouvelle génération de manuels de français en Allemagne.

20 C'est un manuel pour le français deuxième ou troisième langue dans les lycées allemands. Il se distingue des manuels en usage à l'époque notamment par les points suivants :

21 - Il est centré sur le vocabulaire du FF1 (qu'il enseigne fidèlement, en ajoutant un certain nombre de mots hors FF1).

22 - Il demande une approche orale interactive.

23 - Cette approche est facilitée par un support visuel en forme de " cartes murales » pour chaque leçon.

24 - Les 30 leçons représentent chacune une unité thématique (sur le contenu « culturel» cf. Barrera-Vidal 1972).

25 - Les leçons présentent une suite de textes, en général en forme de dialogues ou de questions-réponses, accompagnés d'exercices de vocabulaire et de grammaire et d'explications grammaticales.

26 - Quant à la grammaire, les auteurs (linguistes de formation et de profession) proposent une approche contextuelle et contrastive (Franke 1972).

\subsection{Salut en classe de langue : les conseils méthodologiques de ses auteurs}

Selon l'intention des auteurs du manuel, le professeur est censé commencer chaque leçon par l'exploitation d'une carte murale (qui est reproduite dans le manuel même). L'élève se familiarise, par ces dessins, avec le sujet traité dans la leçon et il prend connaissance, par la voix du professeur et le support visuel, d'une partie du vocabulaire. Les textes qui seront ensuite présentés par le professeur, servent comme base de l'interaction en classe et pour le travail à la maison. Il y a quelques textes narratifs, qui invitent à la lecture.

La plupart des exercices, qui se réfèrent aux textes des leçons, se prêtent au travail oral, quelques-uns au travail écrit. A la fin de chaque leçon, on trouve une liste bilingue des mots nouveaux, parfois organisée en champs lexicaux et en familles de mots. Une liste supplémentaire contient des expressions, des locutions et des collocations fréquentes du vocabulaire enseigné, souvent des proverbes. A la fin du manuel, il y a 
une liste complète du vocabulaire par ordre alphabétique. Elle contient tous les mots du FF1, les mots qui vont au-delà de ce vocabulaire sont indiqués.

\subsection{Les sujets des leçons}

Les sujets des leçons concernent la vie de tous les jours, comme elle se présente à un jeune voyageur allemand dans les années 1960 en France : la vie familiale, des activités de tous les jours (faire des achats, aller à la banque, etc.), la vie des jeunes (les loisirs, fêter un anniversaire), le choix d'un métier, des voyages en France (à la recherche d'un hôtel, les auberges de jeunesse). Les voyages servent à présenter des faits de civilisation et des informations historiques. A l'intérieur des dialogues, on parle de temps en temps de textes littéraires ${ }^{7}$. Les auteurs ouvrent parfois une fenêtre sur d'autres sortes de textes : bulletins radiophoniques, publicité, lectures des jeunes, proverbes $^{8}$, « histoires drôles ».

\subsection{Un vocabulaire FF1 élargi}

Salut présente - on l'a déjà dit- le vocabulaire du FF1 in extenso. Certains critiques auraient préféré que les auteurs se limitent à la partie fréquente de ce vocabulaire, en laissant de côté la partie « disponible » (voir Stammerjohann 1983).

Barrera-Vidal et Franke (1968a) ont enrichi cependant le vocabulaire du FF1 : ils veulent compléter des familles de mots ( $\mathrm{p}$. ex. acheter (FF1) par achat (FF2)), arrondir certains champs lexicaux (p. ex. le champ « famille » est complété par beau-père et bellemère, le champ " repas " par baguette et croissant), compléter des domaines dans l'intérêt du public jeune (dans le domaine " hôtel " on ajoute auberge de jeunesse, ajiste et camping, dans le domaine « loisirs » on ajoute football, bricolage, match, nage, maillot de bain, piscine, guitare, ski, tennis, tourne-disque), compléter les connaissances sur la civilisation contemporaine (on enseigne à côté d'industrie (FF1) les termes agriculture, économie et industrie atomique (FF2)), introduire dans l'histoire : château, roi, reine, Renaissance, Révolution. Ils enseignent enfin un vocabulaire technique pour l'apprentissage des langues (le discours de la salle de classe, la terminologie grammaticale, un vocabulaire de base pour des entretiens sur la littérature, en vue du travail dans les phases ultérieures de l'enseignement du français).

F. Abel (1984) regrette qu'ils n'aient pas suffisamment contextualisé ce vocabulaire. Une seule contextualisation (comme on la trouve en effet souvent dans les leçons) ne suffit pas selon lui pour ce vocabulaire souvent polysémique. Or Abel n'a étudié que les textes des leçons sans prendre note des exercices et des listes des mots en fin des leçons qui témoignent d'un emploi plus riche du vocabulaire

\subsection{Les codes : l'oral et l'écrit}

La décision de composer un manuel essentiellement de dialogues et de le concevoir comme «interactif» est sans doute fidèle à l'esprit du Français fondamental. Elle reflète aussi l'influence des méthodes audio orales et audio-visuelles. Or, quant à l'emploi de la langue parlée, Salut est moins puriste que le Français fondamental. On y trouve des expressions supprimées dans les listes du FF1 - malgré leur haute fréquence - comme ah, oh, bah, zut, salut !, ainsi que des termes familiers comme bouquin et bricolage. 
D'autre part, on trouve, dans Salut, des expressions appartenant à un niveau de langue inattendu dans un manuel pour débutants : "Présentez mes hommages à Madame votre mère » dit un jeune Allemand à une jeune Française qui l'a invité à la maison. Au cours de la soirée, on est témoin de scènes de présentation comme "Vous me permettez de vous présenter à ma camarade Jacqueline ? " et de la réponse " Je suis heureuse de faire votre connaissance, Mademoiselle $»^{9}$.

Le recours à des éléments du FF2 élargit la part du code écrit dans ce manuel du " niveau I ", voué essentiellement à l'oral. Les auteurs ont l'intention de préparer les apprenants à la lecture et à la production écrite. Dans cette intention, ils introduisent des expressions argumentatives comme quant à, grâce à, par contre ${ }^{10}$.

\subsection{Problèmes qui résultent de la décision d'enseigner le vocabulaire du FF1 dans sa totalité}

Certains champs lexicaux du FF1 sont difficiles à intégrer dans un cours de français pour débutants, si l'on ne veut pas perdre de vue leurs besoins langagiers spécifiques. Voici deux exemples :

$1^{\circ}$ Les animaux. La liste des bêtes est longue dans le FF1, et on en connaît les raisons. On y trouve âne, animal, bête, bøeuf, cheval, chèvre, chien, cochon, coq, insecte, mouche, moustique, mouton, oiseau, poisson, poule, poulet, serpent, vache, veau. Et si l'on ajoute aile, bec, corne, œuf, patte, plume, poil, voler (dans le sens de la locomotion des oiseaux), on arrive à vingthuit entrées pour la ménagerie.

Comment les auteurs de Salut arrivent-ils à intégrer ce vocabulaire sans rédiger une leçon de zoologie ? Ils enseignent les centres d'intérêt " animaux » distribué en divers contextes. En voilà trois exemples : on explique, dans le contexte d'une séance de cinéma, un film publicitaire qui propage la viande de bøuf maigre, dans lequel on montre des scènes de campagne avec des chevaux, des vaches, des veaux et des cochons (leçon 12). Un personnage du manuel - Maurice - aime raconter des histories drôles. « Monsieur, vous êtes un âne ! - Et vous un veau! - Et bien, nous nous retrouverons sur le pré ... demain, à six heures. " Dans la même leçon (leçon 28), on distingue des " histoires de bêtes » et des " histoires bêtes ", ce qui prouve une certaine distance ironique des auteurs à l'égard de leurs propres textes.

A la dernière leçon "Le camping ", il fallait encore enseigner les noms de quatre animaux qu'on n'avait pas mentionnés avant. Voici un court dialogue :

Michel : Ah voilà des invités dont nous pourrions nous passer

Eric : Quoi ? Des mouches?

Michel : Pire que ça, des moustiques! Sales insectes! Ils m'ont déjà piqué au cou.

Eric : Ne t'en fais pas! Tant qu'il n'y a pas de serpents.

$2^{\circ}$ L'agriculture. La leçon 29 contient dix mots du champ lexical « agriculture »: ferme, champ, charrue, herbe, foin, blé, paille, tas, moisson et labourer. Ce vocabulaire est introduit dans le contexte d'un voyage en auto, à travers champs, après la moisson du foin. Le voyage se termine dans un tas de paille, suite à un accident de voiture. On est content de ne pas être rentrée dans la charrue qui avait servi à labourer la terre. Dans la même leçon, l'élève est occupé d'un autre champ lexical, concernant " l'accident ", à savoir médecin, police, assurances, un vocabulaire probablement plus utile aux jeunes 
touristes que celui de l'agronomie d'antan. Dans la leçon 30, l'action de « monter une tente » dans un terrain de camping est égayée d'une émission de radio sur l'agriculture et l'industrie en France. Cet arrangement permet l'introduction des champs lexicaux " les outils de travail " (le marteau, la manche, la pioche, la pelle, le tracteur), et « le travail dans les champs " (semer, creuser, planter, récolter, le grain) et de parler en même temps de l'industrie moderne (pétrole, puits de pétrole, énergie [FF2], force de frappe $[$ frapper $=$ FF1 $]$ ).

\subsection{Le pour et le contre d'un vocabulaire de base pour tous} commun, qui pourrait servir à harmoniser les programmes du premier cycle de l'enseignement secondaire comme « vocabulaire obligatoire pour tous» (Christ 1970). Or, ils sont restés plus ou moins seuls avec cette bonne intention. Les autres manuels ne les ont pas suivis dans cette voie (cf. Abel 1984, Möhle 1980), ni d'ailleurs les programmes officiels ${ }^{11}$. Nous citons Raasch (1972) et Nickolaus (1972) comme témoins de la critique contre un "vocabulaire obligatoire pour tous " Raasch (1969a) est l'auteur d'un Mindestwortschatz (vocabulaire de base) à l'usage des Volkshochschulen qui contient 1500 mots. Il est composé de mots choisis du FF 1 et du FF2, auxquels des " experts allemands et français " (Préambule) ont ajouté d'autres mots pour les besoins spécifiques du public. On a écarté du lexique FF 1 - pour donner quelques exemples - les centres d'intérêt " agriculture ", « élevage des bêtes » et " métiers artisanaux », et on a adopté des mots du centre d'intérêt « voyage » (comme : caméra, auberge de jeunesse, camping, guichet, carte d'identité, parking, station de service, stop, wagon-restaurant.)

Le Mindestwortschatz présente les mots avec leurs équivalents allemands (en général plusieurs par entrée), mais aussi des collocations, des phrases utiles, des remarques sur la prononciation. En voici quelques exemples pour les collocations et les phrases utiles : le mot grammatical à (dans la terminologie de Raasch un Strukturwort) se trouve dans les collocations suivantes : à Paris, à dix heures, à midi, à demain, à tout à l'heure, au printemps, à dix kilomètres d'ici, c'est facile à faire, c'est à vous, à côté, à côté de, à droite (de), à gauche (de), j'écris une lettre à mon frère. Et pour le mot carte on présente quatre synonymes, dont trois du moins sont d'utilité pour le touriste : jouer aux cartes, manger à la carte, carte d'identité, carte postale. Le choix du vocabulaire (qui doit servir des adultes de langue allemande en situation de contact avec des francophones, notamment en situation de voyage, dans les échanges et les rencontres) est donc fait selon les besoins supposés des apprenants.

Nickolaus (1961) a élaboré, à l'usage des apprenants dans les écoles, un vocabulaire divisé en deux parties : un Grundwortschatz (vocabulaire de base) de 2000 mots (auquel s'ajoutent 75 " mots grammaticaux »), et un Aufbauwortschatz (vocabulaire progresssif ou du Niveau II) de 2500 mots. Le Grundwortschatz est présenté par ordre alphabétique, le Aufbauwortschatz est divisé en 72 centres d'intérêt (par exemple : Fehler, Laster ${ }^{12}$ ) ou Religion, religiöses Gefühl' ${ }^{13}$ ), groupés en 13 chapitres (pour le cas présent : Seelen- und Gefühlsleben ${ }^{14}$ ). Chacun des centres d'intérêt reprend en exergue les mots du Grundwortschatz qui correspondent à sa thématique. L'auteur complète la liste des mots (et leurs équivalents allemands) par des locutions et des phrases qui remettent les mots dans différents contextes. 

Français fondamental d'environ 1000 mots. L'auteur a élargi les listes de 1600 unités, après avoir supprimé environ 100 unités du FF1 et 500 unités du FF2, donc au total 600 mots des deux degrés du Français fondamental. Un bon tiers des mots ne provient ni du FF1 ni du FF2. Dans une analyse détaillée, I Christ (1970) a montré que le vocabulaire du Grund- und Aufbauwortschatz Französisch privilégie la langue littéraire, donc la langue écrite. Ceci s'annonce déjà dans le Grundwortschatz, où la langue littéraire occupe une place importante, au désavantage de la langue parlée. Comme Raasch, Nickolaus (1972) justifie sa sélection par les besoins des apprenants, dans son cas les élèves du lycée. La raison de leur refus d'un « vocabulaire obligatoire pour tous » est donc la même : le vocabulaire enseigné doit être conforme aux besoins des apprenants et à leur curriculum spécifique.

\subsection{Enseigner la grammaire du Français fondamental ou non}

On sait que Gougenheim et ses collaborateurs ont formulé, concernant la grammaire, certains principes, et ils ont rassemblé des éléments pour une future grammaire sous forme de prescriptions introduites de façon stéréotype par la formule « on enseignera/on n 'enseignera pas " (voir le chapitre "La Grammaire " dans "L'Elaboration ", Gougenheim et al. 1964: 211-230). Ces prescriptions se basent partiellement sur des enquêtes statistiques préalables.

Les auteurs de Salut ont suivi ces conseils à leur manière : ils ont rédigé, à l'usage des apprenants, un cahier grammatical \{Grammatisches Beiheft, Barrera-Vidal et Franke 1968b) qui se veut élémentaire au sens d'exposer et d'expliquer le contenu grammatical du manuel leçon par leçon, texte par texte, sans aspirer à présenter une "grammaire fondamentale systématique $»^{15}$. Ils se limitent par principe au contexte présenté à l'apprenant. Le cahier est donc uniquement conçu pour les utilisateurs de Salut qui apprennent la grammaire dans le contexte des leçons de leur manuel.

51 Ainsi, dans la première leçon, les apprenants font connaissance des articles (indéfinis et définis) au singulier, et des pronoms il et elle -phénomènes grammaticaux qu'ils peuvent découvrir eux-mêmes, de façon "inductive ", et qu'ils retiennent à l'aide du professeur comme "régularités ", notées au cahier telles quelles. Tout le reste du contenu langagier de la leçon est "lexique ». Les noms sont présentés uniquement au singulier, les verbes auxiliaires être et avoir uniquement à la troisième personne du singulier.

52 La distinction entre singulier et pluriel est le sujet de la $2^{\text {eme }}$ leçon. On y introduit aussi la terminologie correspondante. Mais dans ce cas aussi, on ne pense pas encore à compléter la conjugaison des verbes. On se contente des $3^{\text {emes }}$ personnes. On enseignera les autres au fur et à mesure de leur apparition dans des textes, et selon les besoins communicatifs des apprenants. Le procédé est par principe contextuel, la méthode inductive, l'intention communicative.

Or, il y a des phénomènes grammaticaux qui demandent une explication systématique, surtout dans les cas d'un fort contraste entre l'allemand et le français. Nous prenons comme exemple le subjonctif (leçon 27) qui pose des problèmes spéciaux pour les apprenants de langue allemande, vue que le Konjunktiv allemand a des fonctions différentes.

Documents pour l'histoire du français langue étrangère ou seconde, 36 | 2006 
Dans le chapitre La grammaire (Gougenheim et al. 1964 : 221) on lit sur le subjonctif :

(58) On n'enseignera le subjonctif (présent et passé) que dans certaines tournures limitées après les expressions : avant que, pour que, avoir peur que, il faut que, vouloir que.

Le cahier se contente d'abord du subjonctif présent. Or les régularités de son emploi sont longuement expliquées. On distingue entre l'emploi obligatoire du subjonctif (les cas de la " servitude grammaticale ») et son emploi facultatif, à nuance stylistique. Pour l'emploi obligatoire, Barrera-Vidal et Franke présentent plus de 20 "tournures " (contre les 5 cités dans la grammaire). Cette longue liste est en effet importante pour des apprenants de langue allemande qui attendent l'indicatif, selon leurs habitudes en langue maternelle, après des tournures comme ils veulent que, il se plaint que, je n'aurais pas pensé que. On constate donc que le cahier part d'un point de vue contrastif.

\section{En guise de conclusion : du Français fondamental à «Un Niveau-seuil »16}

Nous avons voulu montrer jusqu'à quel point l'enseignement du français en Allemagne a été influencé et transformé par le Français fondamental. Il a mené en effet à une nouvelle discussion sur la progression. Son influence était très forte dans le domaine de la sélection et $\mathrm{du}$ choix du lexique. Elle était moins forte dans le domaine de la grammaire, pour des raisons que nous avons pu montrer ${ }^{17}$. Nous savons, d'autre part, que le Français fondamental a divisé l'opinion des didacticiens en trois points importants :

57 - quant au rôle et à la fonction de la langue parlée dans la classe de langue,

58 - quant à l'appréciation des besoins langagiers des apprenants,

59 - et enfin quant à la définition d'un vocabulaire obligatoire qui pourrait servir à l'harmonisation du curriculum pour les classes de débutants.

60 La discussion a pris un autre tournant au moment où les travaux du Conseil de l'Europe - notamment concernant The Threshold Level, Un Niveau-seuil et Kontaktschwelle Deutsch (cf. van Ek 1977) - sont entrés en scène. Pour mettre en relief ce changement, citons un article de L. Franke publié en 1977, presque dix ans après la publication de Salut. En 1977, Franke ne part plus, pour définir les objectifs de la classe de débutants, (fidèle à la position du Français fondamental), de la langue mais il essaie de décrire le profil de l'apprenant à la fin du premier cycle, pour déduire de ce profil de l'apprenant les contenus langagiers de l'enseignement du français.

61 Ceci le mène à définir les rôles et les situations dans lesquels l'apprenant devra agir, les thèmes qui l'intéresseront, les intentions qu'il voudra exprimer. C'est en fonction de ces facteurs qu'il faudra, selon lui, déterminer les moyens langagiers pour la communication orale et écrite, et donc le contenu de l'enseignement ${ }^{18}$.

62 Comment se présente, sous ces nouveaux aspects, l'idée de la " langue fondamentale » ? Le Français fondamental peut-il encore servir ? On sent dans cet article de Franke un certain malaise. Il ne désavoue pas ce qu'il a dit 10 ans avant. Mais il propose maintes modifications et révisions dans l'utilisation du Français fondamental. Nous le montrerons à l'exemple du lexique. 
63 $d u$ FF1. Son vocabulaire «thématique» sera par contre celui de ses besoins langagiers, qu'il trouvera - si tout va bien -dans les thèmes et les situations de son manuel et non plus dans le vocabulaire disponible du FF1. Il ne peut donc plus être question d'un vocabulaire de base obligatoire pour tous.

Au cours du premier cycle, l'élève apprendra un « vocabulaire de la définition ». On lui enseignera " les lois de la formation des mots ", afin qu'il puisse élargir de façon autonome son vocabulaire potentiel. Il apprendra, dès le début, pas à pas, les mots grammaticaux non pas seulement du FF1, mais aussi du FF2. L'idée directrice de cette nouvelle vue sur le lexique est d'individualiser le curriculum.

C'est donc l'apprenant qui sera à la base de toutes les réflexions et toutes les décisions didactiques. Nous avons à faire à un changement de paradigme en didactique : après la langue c'est l'apprenant qui sera au centre de la réflexion. Ceci était en effet la devise du Congrès de la FIPLV (Fédération internationale des professeurs de langue vivantes) à Sarrebruck en 1972 : Focus on the learner.

\section{BIBLIOGRAPHIE}

ABEL, F. (1984), «Der französische Häufigkeitswortschatz nach Juilland in zwei verbreiteten Lehrwerken für den Französischunterricht in Deutschland ", Theoretische und praktische Probleme der Lexikographie, Goetz D., Herbst Th. (éds)., München : Hueber, pp. 18-49.

BAKONU, H. (1934), Die gebräuchlichsten Wörter der deutschen Sprache, für den Fremdsprachenunterricht zusammengestellt. München.

BARRERA-VIDAL, A. (1972), « La place et la fonction de la civilisation française dans un manuel de français : Salut et l'information culturelle ", Französisch heute 4, pp. 64-68.

BARRERA-VIDAL, A. (1977), «Zur didaktischen Begründung der Sprachminima für den Spanischunterricht » Sprachminima und Abschlussprofile, Hüllen, Raasch, Zapp (éds),, pp. 21-28.

BARRERA-VIDAL, A. (1979), «Un vocabulaire de base orienté sur les besoins des apprenants? Quelques observations sur le 'Grundbaustein Französisch' », Zielsprache Französisch, pp. 82-86. BARRERA-VIDAL, A. et FRANKE, L. (1968a), Salut. Französisches Lehrbuch. Grundkurs, Frankfurt am Main : Diesterweg.

BARRERA-VIDAL, A. et FRANKE, L. (1968b), Salut. Grammatisches Beiheft. Frankfurt am Main : Diesterweg.

BETZ, W. et STAMMERJOHANN, H. (1975), «Grundwortschatz» Handbuch der Linguistik. Allgemeine und angewandte Sprachwissenschaft, Stammerjohann H. (éd), München :Nymphenburger, pp. 184-186.

CHRIST, I. (1970), «Ein verbindlicher Grundwortschatz für den Französischunterricht? » Praxis des neusprachlichen Unterrichts 19, pp. 32-40.

Documents pour l'histoire du français langue étrangère ou seconde, 36 | 2006 
DEBYSER, F. (1970), « L'Enseignement du français langue étrangère au Niveau 2 », Le Français dans le Monde 73, pp. 6-14.

Deutscher Volkshochschul-Verband e.V., (1979), Das VHS-Zertifikat Französisch., Bonn.

FRANKE, L. (1972), «Die direkte Frage des Französischen im Unterricht », Die Neueren Sprachen 71, pp. 505-520.

FRANKE, L. (1977), «Abschlussprofile der Sekundarstufe I für Französisch » Sprachminima und Abschlussprofile, Hüllen, Raasch, Zapp (éds), pp. 104-118.

GOUGENHEIM, G. et al. (1964), L'Elaboration du Français fondamental (Ie' degré). Etude sur l'établissement d'un vocabulaire et d'une grammaire de base. Paris : Didier.

HÜLLEN, W. et al. (éd), « Sprachminima und Abschlussprofile », Frankfurt am Main : Diesterweg.

KAEDING, F. W. (1898), Häufigkeitswörterbuch der deutschen Sprache, Steglitz.

KAUFMANN, G. (1977), «Die Gewinnung lexikalischer und grammatischer Minima als linguistisches und didaktisches Problem » Sprachminima und Abschlussprofile, Hüllen, Raasch, Zapp (éds), pp. 48-70.

KULTUSMINISTERIUM DES LANDES Nordrhein-Westfalen (éd.) (1973), Curriculum Gymnasiale Oberstufe Französisch, Düsseldorf.

MICHÉA, R. (1966), Grammaire de l'allemand fondamental. Paris : Didier.

MÖHLE, D. (1980), Die Wortschatzstruktur in Lehrwerken für den Französischunterricht. Untersucht am Beispiel des Cours de base, Frankfurt am Main : Lang.

NICKOLAUS, G. (1961), Grund- und Aufbauwortschatz Französisch, Stuttgart : Klett.

NICKOLAUS, G. (1972), «Noch einmal: ,Ein verbindlicher Grundwortschatz für den Französischunterricht'?» Praxis des neusprachlichen Unterrichts 19, pp. 327-328.

OEHLER, H. (1972), «Der mehrsprachige Grundwortschatz als Lern-und Lehrhilfe auf dem Weg zur Mehrsprachigkeit », Der fremdsprachliche Unterricht 23, pp. 2-12.

PELZ, M. (1972), «Frequenzforschung und Fremdsprachenunterricht» Der fremdsprachliche Unterricht 23, pp. 33-43.

PFEFFER, J. A. (1969), «Grunddeutsch», Zeitschrift för deutsche Sprache 25, pp. 132-141.

PUREN, Ch. (1988), Histoire des méthodologies de l'enseignement des langues, Paris : CLE International.

RAASCH, A. (1969a),- Französischer Mindestwortschatz. Mit einem Anhang: Grammatische Grundstrukturen, München : Hueber.

RAASCH, A. (1969b), Das VHS-Zertifikat Französisch, Bonn : Deutscher Volkshochschul-Verband.

RAASCH, A. (1972), «Neue Weg zu einem Grundwortschatz », Praxis des neusprachlichen Unterrichts 19 , pp. 235-244.

RAASCH, A. (1977), « Lernzielorientierte Sprachinventare im Französischen » Sprachminima und Abschlussprofile, Hüllen, Raasch, Zapp (éds), pp.71-80.

RAASCH, A. (1978), « Ein neues Français fondamental? - Das Europarat-Projekt "Un niveau-seuil" ", Praxis des neusprachlichen Unterrichts 25, pp. 292-301.

REISKE, H. (1977), «Sprachminima für die Zielsprache Italienisch» Sprachminima und Abschlussprofile, Hüllen, Raasch, Zapp (éds), pp. 81-90. 
STAMMERJOHANN, H. (1983), «Lexik: Grundwortschatzprobleme», Französisch für Lehrer. Linguistische Daten für Studium und Unterricht. München : Max Hueber, pp. 161-212.

STÖTZEL, G. (1970), « Grunddeutsch (Basic German) », Linguistik und Didaktik 1,pp. 195-204.

STOURDZE, C. (1969), « Les Niveaux de langue », Le Français dans le Monde 65, pp. 18-21.

TARNÓCZI, L. (1971), « Wortbestand, Wortschatz, Wortfrequenz » IRAL IX, pp. 297-318.

VANDER BEKE, G. E. (1929), French Word Book New York: Macmillan.

VAN Ek, J. A. (1977), «The Threshold Level. Ein Projekt des Europarats » Sprachminima und Abschlussprofile, Hüllen, Raasch, Zapp (éds), pp. 91-103.

\section{NOTES}

1. Selon la terminologie de C.Puren (1988).

2. En République fédérale d'Allemagne, il n'y a pas de programmes officiels nationaux, mais des programmes au niveau des Länder.

3. Voir Le Français dans le Monde, 73 (1970), et notamment l'introduction de F. Debyser dans ce numéro thématique.

4. Les recherches sur le "Grunddeutsch » - l'allemand fondamental - (dans des travaux de chercheurs américains et européens) se réfèrent constamment au Français fondamental.

5. Tarnöczi entend par « Basiswortschatz » grosso modo les « mots grammaticaux ».

6. Par exemple par observations de classes par vidéo

7. On cite Camus et Ionesco

8. Sur le rôle et la place de la civilisation dans Salut voir Barrera-Vidal (1972)

9. «Hommage " ne figure pas dans le FF1. On imagine que les auteurs ont voulu sensibiliser les jeunes à différents niveaux de langue, ici la « langue soignée » (Stourdzé 1969) de la classe moyenne

10. Nous ne nous occuperons pas ici du chapitre Vers la lecture, qui est placée en appendice, après les trente leçons, dans lequel les auteurs présentent des extraits de textes littéraires authentiques dans l'intention de servir de passages au Niveau II.

11. A une exception près: Hessischer Kultusminister (éd. 1980), Rahmenrichtlinien - Sekundarstufe I - Neue Sprachen, Frankfurt am Main : Diesterweg

12. Les défauts et les vices

13. La religion et le sentiment religieux

14. L'âme et les sentiments

15. P. Rivenc a attiré notre attention sur la Grammaire de l'Allemand fondamental de R Michéa (Michéa 1966). Michéa est l'auteur du chapitre «La grammaire » dans l'Elaboration. Dans l'ouvrage de 1966 il montre en effet - à l'exemple de l'allemand - comment on aurait pu écrire une grammaire fondamentale du français systématique, limitéed'une part aux parties du discours et d'autre part à l'ordre des mots dans la phrase. Le Grammatisches Beiheft de Barrera-Vidal et Franke est conçu dans unesprit tout à fait autre.

16. Voir Raasch 1978.

17. On peut même dire que le Français fondamental a influencé la didactique et la pratique de l'enseignement d'autres langues, comme par exemple de l'allemand langue étrangère (Kaufmann 1977, Oehler1972, Pfeffer 1969, Stötzel 1870 ; voir aussi Michéa 1966).

18. Voir Raasch 1972 
INDEX

Mots-clés : français fondamental, Allemagne, vocabulaire de base, Niveau-seuil, matériaux didactiques

Keywords : basic French, basic vocabulary, Germany, Niveau-Seuil, didactic materials

\section{AUTEURS}

HERBERT CHRIST

Düsseldorf (Allemagne

INGEBORG CHRIST

Düsseldorf (Allemagne) 